\section{Asian medicine: Japan's paradigm}

The international medical community could benefit from the wide range of therapeutic options that traditional Japanese Kampo medicine can offer. Its integration into modern medicine has already been realized in Japan (Nature 480, S96; 2011), where it is available as a 5 -year specialization for physicians already trained in Western medicine.

Kampo and traditional Chinese medicine have common roots, but Kampo uses additional diagnostic techniques and more rigorously controls the quality of herbal preparations.

It would be a major loss for both Western and traditional medicines if political or financial factors were to cause the "sun to set" on Kampo.

Silke Cameron University Medicine Göttingen, Germany. silke.cameron@med.unigoettingen.de

Heidrun Reissenweber Clinic for Japanese Medicine, Munich, Germany.

Kenji Watanabe Center for Kampo Medicine, Keio University School of Medicine, Tokyo, Japan.

\section{Asian medicine: a fungus in decline}

Estimates of wildlife trade for traditional Asian medicine should include that of the caterpillar fungus Ophiocordyceps sinensis (Nature 480, S101-S103; 2011).

The fungus, used to treat asthma and other diseases, is legally harvested on a huge scale in Tibet and the Himalayas, and is one of the world's most expensive natural medical resources. Some 85-185 tonnes are collected annually by the local population for a global market worth between US $\$ 5$ billion and $\$ 11$ billion.

Large increases in the price (up by $900 \%$ from 1997 to 2008) and trade of caterpillar fungus have encouraged more intensive harvesting. My informal survey of harvesters in the Himalayas reveals that caterpillar fungus abundance is dwindling: the average harvest per collector dropped by around half between 2006 and 2010. Harvesters are extending their range as a result, risking overexploitation of a pristine landscape and more ecosystem degradation.

Conservation efforts must be initiated to halt the decline of this species, which is causing a loss of biodiversity and threatening local livelihoods.

Uttam Babu Shrestha University of Massachusetts, Boston, USA. ubshrestha@yahoo.com

\section{Asian medicine: many unique types}

The different branches of traditional Asian medicine are frequently confused (Nature 480, S81-S103; 2011). Now could be the time to revive the centuries-old term 'Eastern medicine' to avoid such inaccuracies and to complement descriptions of Western medicine.

'Oriental' and 'Asian' medicine collectively describe the range of traditional treatments used in many Asian countries. Traditional Chinese medicine is more specific. Although practised mainly in China, it influenced the development of traditional medicines unique to Japan, Korea and Vietnam in the past few hundred years. Lumping all of these together as 'traditional Chinese medicine' is therefore incorrect.

The term 'Eastern medicine' was first coined in 1613 by a court physician in Korea, Heo Jun, in his book Donguibogam ('Principles and Practice of Eastern Medicine'). The book is still used in clinics and, in 2009, was added to the United Nations Educational, Scientific and Cultural Organization's World Documentary Heritage list.
Hoyun Lee Health Sciences North, Sudbury, Ontario, Canada.mhlee@hsnsudbury.ca

\section{Asian medicine: call for more safety data}

Marketing of traditional Chinese medicines is developing rapidly worldwide (Nature 480, S81S103; 2011). So much so that the European Union (EU) issued a directive in 2004 that all herbal preparations should be subject to the same screening procedures as pharmaceuticals by 2011. But by April last year, no Chinese herbal medicines had met the directive's requirements. Many have therefore been withheld from sale in the EU.

If these traditional remedies are to be accepted, their quality needs to be standardized and rigorous scientific data must be supplied on their efficacy and safety. The mystique surrounding such treatments must give way to verification and a proper understanding of concepts and applications. Only then can traditional Chinese medicine be integrated into a global health-care system. Juncai Xu, Min Liu Shanghai Clinical Research Center, Shanghai, China.

Zhijie Xia Shanghai Huashan Hospital, Fudan University, China.janexia2006@126.com

\section{Asian medicine: protect rare plants}

As the global market in traditional Chinese medicines expands, many wild plants are on the brink of extinction (see also Nature 481, 265; 2012). Urgent measures must be taken to ensure that these rare plants are harvested sustainably.

Some 11,000 plant species are listed in the Chinese pharmacopoeia, medicinal botany textbooks and ancient Chinese medical texts such as the Compendium of Materia Medica and Shennong's Classic of Materia Medica.

Examples of critically depleted natural populations include Herba epimedii, a herb used as an aphrodisiac, tonic and antirheumatic in China, Korea and Japan; Panax ginseng, a tonic and sleep-inducer; Euchresta japonica, for anti-tumour activity; Dysosma versipellis, a cleanser of toxins; and Aconitum brachypodum, an antiinflammatory.

Hua-Feng Zhang Shaanxi Normal University, Xian, Shaanxi, China.

Xiao-Hua Yang Xian Jiaotong University, Xian, Shaanxi, China; and Moscow State University, Russia.yxh@bk.ru

\section{Safety-test initiatives for nanomaterials}

Your report on the need to establish safety regulations for nanomaterials focuses largely on US initiatives (Nature 480, 160-161; 2011). Other initiatives are also making important contributions.

The Organisation for Economic Co-operation and Development (OECD) provides guidance on what parameters should be used for reporting the safety testing of nanomaterials (see go.nature. com/yiaxnd). Projects set up to aid implementation of Europe's REACH (for 'registration, evaluation, authorisation and restriction of chemicals') legislation advise on how to review information on nanomaterials.

The European Food Safety Authority published guidance last year on risk assessment of nanotechnologies in the food chain (see go.nature.com/7131fo). The European Commission's Joint Research Centre has also set up a repository of representative nanomaterials samples.

Juan Riego-Sintes European Commission Joint Research Centre, Institute for Health and Consumer Protection, Ispra, Italy. juan.riego-sintes@ec.europa.eu 